\title{
FREQUENCIA DAS ONDAS CEREBRAIS: UMA PERSPECTIVA DA NEUROCIÊNCIA
}

\author{
Bruna Araújo Caimar ${ }^{1}$
}

Mestranda em Saúde Coletiva com ênfase em Neurociências.

Gabriel César Dias Lopes ${ }^{2}$

Orientador

\section{RESUMO}

As ondas cerebrais frequências causadas por atividades sinápticas que ocorrem no sistema nervoso, geralmente as ondas cerebrais são medidas em ciclos por segundo ou $\mathrm{Hz}$ ou hertz, e são elas classificada em cinco: alfa, beta, gamma, theta e delta. Estas ondas estão ligadas diretamente aos sentimentos aos estados de picos emocionais, estado de distúrbios psicológicos e estados meditativos. Esta pesquisa tem como principal objetivo estudar as ondas cerebrais, seus fluxos e suas variáveis de acordo com as emoções humanas e de acordo com os estados de meditação, relaxamento e picos de estresse e aleta, além de compreender como o cérebro e toda sua estrutura pode produzir e emitir estes sinais e frequências. Para a realização do estudo foi utilizado pesquisas de revisão de literatura, nas bases de dados Scielo, Lilacs, Google Scholar, PubMed, Medline, sendo discutido temas pertinentes ao assunto.

Palavras-chaves: Eletroencefalograma (EEG), ondas cerebrais, emoções humanas.

\section{FREQUENCY OF BRAIN WAVES: A NEUROSCIENCE PERSPECTIVE}

\section{Abstract}

The brain waves frequencies caused by synaptic activities that occur in the nervous system, generally the brain waves are measured in cycles per second or $\mathrm{Hz}$ or hertz, and they are given in five: alpha, beta, gamma, theta and delta. These waves are directly linked to feelings of emotional peak states, state of 
psychological disorders and meditative states. This research has as main objective to study how brain waves, their flows and their variables according to human emotions and according to the states of meditation, relaxation and stress and fin points, in addition to understanding how the brain and all its structure can produce and emit signals and frequencies. To carry out the study, literature review surveys were used in the databases Scielo, Lilacs, Google Scholar, PubMed, Medline, and topics relevant to the subject were discussed.

Keywords: Electroencephalogram (EEG), Brain Waves, Human Emotions.

1 Fisioterapeuta - Logos University International - UNILOGOS. E-mail: brunacaimar@gmail.com 2 Reitor UNILOGOS - E-mail: president@unilogos.education

\section{INTRODUÇÃO}

O cérebro humano é composto por dois sistemas, o sistema nervoso central e o periférico, onde o central consiste no cérebro propriamente dito e na medula e o periférico nos gânglios e nas raízes nervosas. Essas estruturas são o que controlam todo o corpo humano, tanto no movimento, quanto nas emoções, memória, discernimento, etc (KOUDELKOVÁ e STRMISKA, 2018).

O cérebro em sua totalidade tem como função comandar o corpo por inteiro através de estímulos elétricos que enviam e recebem informações através dos neurônios para que a ação desejada venha a ser executada. Os neurônios geram sinais elétricos e esses sinais podem ser medidos através de um exame que se chama eletroencefalograma.

O eletroencefalograma é classificado conforme a frequência das ondas, conforme a amplitude, forma, posicionamento que os eletrodos são colocados no couro cabeludo, podendo estas ondas variar de 0,1 a $100 \mathrm{HZ}$. As ondas são medidas em ciclos por segundo ou $\mathrm{HZ}$, mudando de acordo com as sinapses ou atividades elétricas que o cérebro emite, relacionado estas com estados de alerta, sonhos, sono, mediação, ansiedade, raiva, entre outras (MUNIAN; SIVALINGAM; JAYARAMAN, 2014)

Existem estudos que divergem em alguns aspectos quanto aos $\mathrm{HZ}$ das ondas cerebrais, variando em questão de meio $\mathrm{HZ}$ de uma pra outra. As ondas Beta alcançam e de 14 a $40 \mathrm{HZ}$ e estão associadas a raciocínio, cognição, atenção; Já as ondas Alfa alcançam de 7.5 a $14 \mathrm{HZ}$ e está relacionada com a 
criação, relaxamento e meditação; A onda Theta alcança de 4 a $7.5 \mathrm{HZ}$ e tem relação com a criação memoria, quando a pessoa está dormindo; A onde Delta alcança de 0.5 a $4 \mathrm{HZ}$ e está relacionada com consciência, recuperação ou cura, sono profundo; e por fim a onda Gamma alcança acima de $40 \mathrm{HZ}$ e está relacionada com os insights (ISMAIL et al., 2018; PASRI, 2019; FONTOURA, 2017).

Esta pesquisa tem como principal objetivo estudar as ondas cerebrais, seus fluxos e suas variáveis de acordo com as emoções humanas e de acordo com os estados de meditação, relaxamento e picos de estresse e aleta, além de compreender como o cérebro e toda sua estrutura pode produzir e emitir estes sinais e frequências. Para a realização do estudo foi utilizado pesquisas de revisão de literatura, nas bases de dados Scielo, Lilacs, Google Scholar, PubMed, Medline, sendo discutido temas pertinente ao assunto. Foram utilizadas palavras chave em português e inglês sendo elas: Eletroencefalograma (EEG), ondas cerebrais, emoções humanas.

\section{CÉREBRO HUMANO}

A capacidade do ser humano de aprender, de discernir, de julgar e de comportar diante de várias situações é possível por conta do encéfalo que é a principal estrutura do sistema nervoso com seus processos de ação e reação (COSENZA e GUERRA, 2011). O encéfalo humano tem como divisão dois hemisférios, direito e esquerdo, que são separados por sulcos ou fissuras. Cada hemisfério é responsável por um conjunto de funções e trabalham no final das contas em conjunto (GAZZANIGA e HEATHERTON, 2005).

O cérebro humano é uma estrutura extremamente complexa, responsável por receber e enviar estímulos, coordenar ações, emoções e gerenciar o corpo como u todo, onde cada área é responsável por uma função, porém que para o pleno funcionamento é necessário a interação entre todas as estruturas e suas funções.

\subsection{NEURÔNIOS}


Existem inúmeros neurônios em nosso encéfalo os quais estão em constante comunicação, mandando e recebendo constantemente impulsos elétricos e estímulos químicos, assim, estas estruturas estão ligadas diretamente á comunicação, emoções, aprendizagem e sofrerem modificações. Os neurônios constantemente disparam impulsos elétricos para gerar e receber informações através das sinapses, onde ocorrem as passagens de informações no sistema nervoso (CAIMAR e LOPES, 2020; COSENZA; GUERRA, 2011).

Os neurônios são estruturas que tem funções especificas dentro do organismo, podendo sofrer estímulos provenientes do meio externo ou até mesmo estímulos advindos do próprio organismo, ocorrendo assim a transmissão de informações para o sistema nervoso central e consequentemente a resposta ao estimulo recebido.

\subsection{ECG}

O eletroencefalograma é uma tecnologia que vem para que haja um registro e monitoramento das ondas cerebrais e para que através dos resultados possam existir estudos e investigações acerca de plasticidade neural cognitiva e afetiva, emoções, estado de meditação, sono, transtornos cognitivos, distúrbios do sistema nervoso, entre outros. O EEG mede as ondas cerebrais de diferentes frequências dentro do cérebro, sendo estas medidas em ciclos por segundo ou HZ (PASRI, 2019).

O eletroencefalograma é uma tecnologia que veio para aprimorar os estudos acerca das ondas cerebrais, correlacionar as atividades cerebrais com estados de raiva ansiedade, depressão, meditação, emoções extremas e casos de meditação e sono profundo.

\section{ONDAS CEREBRAIS}

As ondas cerebrais nada mais são do que atividades impulsionadas pelas correntes elétricas que ocorrem de neurônio para neurônio durante as atividades 
cerebrais. As ondas cerebrais nunca são iguais entre as pessoas, mesmo porque os sentimentos, estados meditativos e os picos de emoção são individual.

As ondas cerebrais são classificadas em cinco classificações, indicando diferentes condições.

\subsection{BETA}

As ondas beta estão na faixa de frequência entre 14 a $40 \mathrm{HZ}$ e elas tem relação com estados de emoções como raiva, medo, estado de alerta, quando a pessoa está ansiosa, ou na correria da vida cotidiana, geralmente a onda que está presente em maior atividade é a beta, assim, quando estamos com um alto nível de estresse diário, consequentemente a frequência beta aumentará.

Segundo Pasri, 2019, as ondas beta correlacionam-se com a consciência normal a qual estamos vivendo no dia-a-dia ou em um estado de alerta mais apurado ou até mesmo raciocínio logico, porém geralmente em nosso cotidiano a onda que mais se destaca são as ondas beta, conforme haja um nível mais elevado de estresse e ansiedade, o nível de ondas beta também irão se elevar.

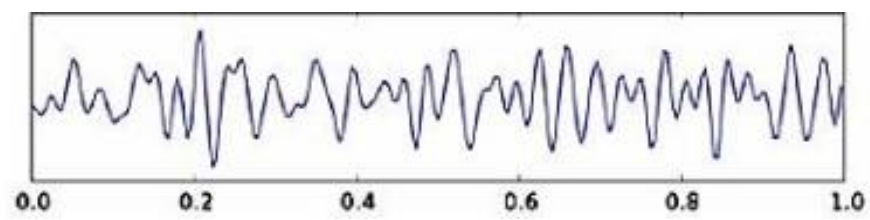

(Adaptado de Kouvelková e Strmiska, 2018).

\subsection{ALFA}

As ondas alfa se caracterizam por estarem presentes e casos de relaxamento, meditação leve, aprendizado e concentração. Geralmente é notado níveis altos de ondas alfas em casos de aprendizagem, momentos criativos, de imaginação e memória (PASRI, 2019; FONTOURA, 2017).

As ondas Alfa tem uma frequência de 7.5 a $14 \mathrm{HZ}$ e essa atividade não tem relação com estímulos sensoriais, mas sim com estímulos visuais, assim, estímulos táteis e sonoros aumentam a frequência alfa. A frequência alfa tem relação direta com aprendizado, com a memória e com a saúde mental. 


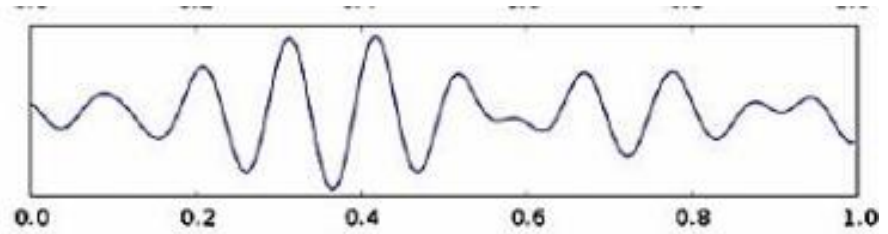

(Adaptado de Kouvelková e Strmiska, 2018).

\subsection{THETA}

A onda Theta alcança uma frequência de 4 a $7.5 \mathrm{HZ}$ e tem relação com estados hipnóticos, de profunda meditação, com o sono, com sonhos, com memorias curtas, quando há momentos reflexivos e quando o individuo tem transtornos depressivos ou de falta de atenção as ondas Theta também são fortemente presentes.

Essas ondas encontram mais presentes em casos de meditação profunda e de baixa consciência, como casos de sono profundo, sonho, hipnose, subconsciente, em casos de quadros depressivos, de impulsividade de hiperatividade (PARSI,2019).

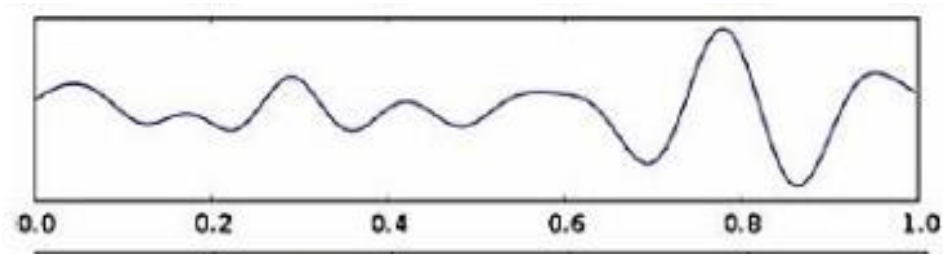

(Adaptado de Kouvelková e Strmiska, 2018).

\subsection{GAMMA}

As ondas Gamma tem sido objeto de recentes estudos, mas as ondas Gamma está correlacionada a estímulos táteis, auditivos e visuais e geralmente tem correlação ao transe, lembranças, meditação, afeto, amor, entre outros.

As ondas cerebrais gama têm uma alta frequência: $40 \mathrm{~Hz}$ a $100 \mathrm{~Hz}$ tem relação a processamento simultâneo de informações em diferentes áreas do cérebro. As ondas Gamma tem ligação com as memórias de curto prazo e 
quanto maior a frequência, mais rápido é possível se lembrar de algo que foi esquecido (PARSI, 2019; FONTOURA, 2017).

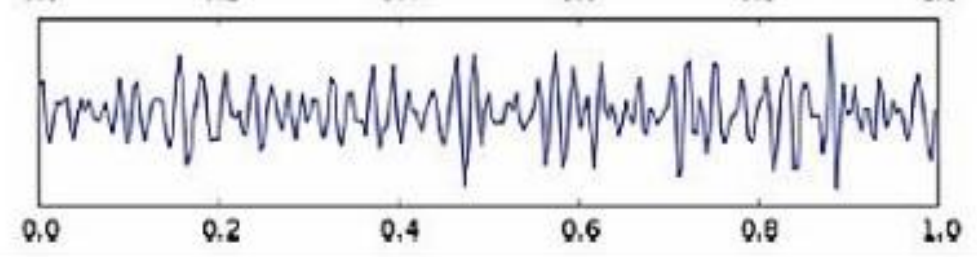

(Adaptado de Kouvelková e Strmiska, 2018).

\subsection{DELTA}

As ondas cerebrais delta têm uma faixa de frequência: $0 \mathrm{~Hz}$ a $3 \mathrm{~Hz}$, são ondas com frequências lentas e estão presentes em somos e meditações profundas, estão presentes em casos de transe e hipnose, em situações de inconsciente e quando estamos com a consciência totalmente inativa. (PARSI, 2019)

Em casos de ondas Delta, quanto maior sua frequência, quer dizer que mais inconsciente ou em estado de inativação da mente o indivíduo estará. Pessoas que conseguem chegar em estados meditativos em frequência delta são pessoas que tem longos anos de experiência, pois é uma frequência muito difícil de ser atingida em estado de consciência.

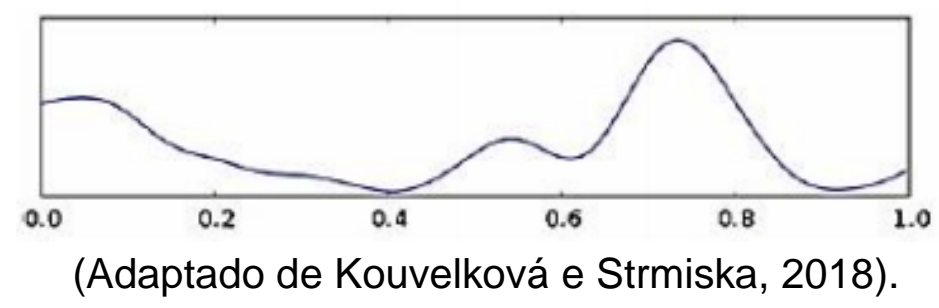

\section{CONCLUSÃO}

Este estudo brevemente pôde analisar as ondas cerebrais, suas frequências e os estados emocionais e meditativos em que cada uma tem sua atuação. As ondas cerebrais são produzidas a partir de atividades neuronais que de acordo com as emoções, sentimentos e atividades de cada indivíduo aumenta ou diminui cada frequência. 
É interessante notar que cada onda quanto maior sua frequência mais agitada se torna a onda e isso tem relação direta com cada estado mental de cada indvíduo. Porém quanto maior a frequência indviual de cada onda, mais característico é cada reação, como por exemplo as ondas delta, que variam de 0 a 3 hertz, quanto maior a frequência de onda, mais característica será as reações do indivíduo com relação ao estado de inconsciência, hipnose, meditação e sono profundo, assim como as ondas beta, que variam de 14 a 40 hertz e está relacionada ao nosso estado de aleta, raciocínio e estado em que vivenciamos no dia a dia, conforme as ondas beta aumentam, significa que estamos mais alertas, mais ativos, com atividades neuronais mais ativas.

São necessários mais estudos acerca das ondas cerebrais para que haja uma melhor compreensão acerca das frequências das ondas neuronais correlacionadas com os estados meditativos, estados de sonolência, sentimentos e picos de emoção. 


\section{REFERÊNCIAS}

CAIMAR, B.; LOPES, G. C. D. Neuroplasticidade: Uma análise da Neurociencia. Revista Cienctifica Cognitions. (DOI): 10.38087/2595.8801.37

COSENZA, R.; GUERRA, L. M e GUERRA. Neurociência e Educação: como o cérebro aprende. Porto Alegre: Artmed, 2011.

FONTOURA, I. Tudo Sobre Neurociências Das Ondas Cerebrais, 2017.

Disponível em: https://hipnosecomneurociencias.com/neurociencias-das-ondascerebrais/

GAZZANIGA, M. S.; HEATHERTON, T. F. Ciência psicológica: mente, cérebro e comportamento. Artmed, 2005.

ISMAIL, O. A. S.; HAMZAH, N.; HANIF, M. Human Emotion Detection Via Brain Waves Study by Using Electroencephalogram (EEG). International Journal on Advanced Science Engineering and Information Technology. v.6, n. 6, 2018.

KOULDELKOVÁ, Z.; STRMISKA, M. Introduction to the identification of brain waves based on their frequency. EDP Sciences. MATEC Web of Conferences. 2018.

MUNIAN, S.; SIVALINGAM, S.; JAYARAMAN, V. Analysis of Real Time EEG Signals. Linaeus University, Chcago, p.1-53, 2014

MARQUES, B. P. Mineração de dados e neurociência: um estudo de sinais cerebrais com base na modulação da amplitude. Dissertação para obtenção do Título de Mestre em Modelagem Computacional, Rio Grande2019.

PASRI, B. Buddhism and brain: beyond the limitation of human brainwaves by buddhist autogenic meditation. The Journal of The International Buddhist Studies College. 2019. 